М. Р. Сайпулаев, И. В. Меркурьев. Динамика и точность микромеханического гироскопа с учетом смещения инерционной массы

Научная статья

УДК 531.383

DOI: $10.18101 / 2304-5728-2020-3-49-62$

\title{
ДИНАМИКА И ТОЧНОСТЬ МИКРОМЕХАНИЧЕСКОГО ГИРОСКОПА С УЧЕТОМ СМЕЩЕНИЯ ИНЕРЦИОННОЙ МАССЫ
}

\author{
(C) Сайпулаев Муса Русланович \\ аспирант, \\ Национальный исследовательский университет «МЭИ» \\ Россия, 111250, г. Москва, ул. Красноказарменная, 14 \\ saypulaevmr@mail.ru
}

\author{
(C) Меркурьев Игорь Владимирович \\ доктор технических наук, доцент, \\ заведующий кафедрой робототехники, мехатроники, динамики и прочности машин \\ Национальный исследовательский университет «МЭИ» \\ Россия, 111250, г. Москва, ул. Красноказарменная, 14 \\ nir4s@ya.ru
}

Аннотация. Рассматривается микромеханический гироскоп, применяемый в системах навигации и управления движением подвижных объектов. В целях повышения точности гироскопа в интегрирующем режиме функционирования на подвижном основании поставлена задача исследования динамики и точности гироскопа с учетом малых инструментальных погрешностей изготовления чувствительного элемента гироскопа - неравной жесткости упругих элементов подвеса, малого смещения центра масс подвижной части конструкции относительно геометрического центра подвеса. Метод повышения точности гироскопа основан на построении новой математической модели динамики и погрешностей гироскопа с использованием общих теорем динамики и асимптотических методов осреднения Крылова - Боголюбова. Построенная новая математическая модель колебаний чувствительного элемента ММГ позволяет оценить погрешности гироскопа в интегрирующем режиме работы в виде зависимости угла прецессии от параметров разнодобротности, разночастотности и смещения инерционной массы на подвижном основании прибора. Приведен сравнительный анализ построенной модели с экспериментальными данными, полученными в случае свободных колебаний чувствительного элемента гироскопа при неподвижном основании. По результатам анализа подтверждена адекватность построенной математической модели ММГ. Для согласования экспериментальных данных с результатами моделирования предложено использовать методики идентификации параметров. Показано, что смещение инерционной массы приводит к изменению собственной частоты колебаний гироскопа и дополнительной разночастотности. Результаты работы могут быть использованы для повышения точности прибора с помощью алгоритма аналитической компенсации погрешности гироскопа.

Ключевые слова: гироскоп R-R типа; прецессия гироскопа; оценка погрешности гироскопа; микромеханический гироскоп; свободные колебания. 


\section{Для цитирования}

Сайпулаев М. Р., Меркурьев И. В. Динамика и точность микромеханического гироскопа с учетом смещения инерционной массы // Вестник Бурятского государственного университета. Математика, информатика. 2020. № 3. С. 49-62.

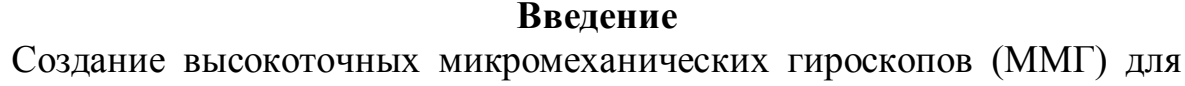
решения задач навигации и управления движением подвижных объектов является актуальной задачей приборостроения [1]. Одним из достоинств ММГ является малая масса и габариты, а также небольшая стоимость (относительно гироскопов, построенных на других физических принципах, таких как, например, волоконно-оптические и лазерные гироскопы). При этом одним из главных недостатков ММГ является нестабильность метрологических характеристик и низкая точность измерений угловой скорости объекта. Принцип функционирования вибрационных гироскопов основан на свойстве маятника Фуко сохранять неизменной в инерциальном пространстве плоскость малых колебаний маятника [2].

Основы теории гироскопов класса обобщенного маятника Фуко и, в частности, ММГ изложены в работах [2-8], где рассмотрены различные схемы построения таких гироскопов, изучено влияние инструментальных погрешностей изготовления, условий функционирования, а также геометрической и физической нелинейностей на точность прибора.

Исследования в этой области нашли отражение и в работах зарубежных авторов [9; 10]. Так в публикации [10] проводится оценка уходов гироскопа на основе построенной математической модели, описывающей медленное изменение амплитуд и фаз колебаний чувствительных элементов (ЧЭ) и элементов орбиты колебаний. В работе [9] обсуждаются проблемы, возникающие при конструировании ММГ, и анализируются уравнения колебаний ММГ. Также в статье [9] составляются уравнения движения для ММГ с угловым и линейным осцилляторным типом движения ЧЭ (R-R и L-L типа, соответственно).

$\mathrm{B}$ целях повышения точности ММГ в данной работе исследуются колебания ЧЭ ММГ R-R типа с учетом явлений разнодобротности и разночастотности, а также эффектов, возникающих из-за смещения центра масс ЧЭ относительно центра карданова подвеса. Смещение центра масс появляется ввиду несовершенства технологии изготовления и сборки прибора. Поставлена задача повышения точности ММГ путем построения новой математической модели колебаний ЧЭ ММГ с учетом указанных выше инструментальных погрешностей и аналитической компенсации ухода гироскопа в линейном приближении.

\section{1 Уравнения колебаний задачи}

Рассматривается конструкция микромеханического вибрационного гироскопа R-R типа с промежуточной рамкой [3]. Кинематическая схема гироскопа представляет собой двухстепенной карданов подвес ЧЭ. Конструкционная схема прибора приведена на рис. 1 . 
М. Р. Сайпулаев, И. В. Меркурьев. Динамика и точность микромеханического гироскопа с учетом смещения инерционной массы

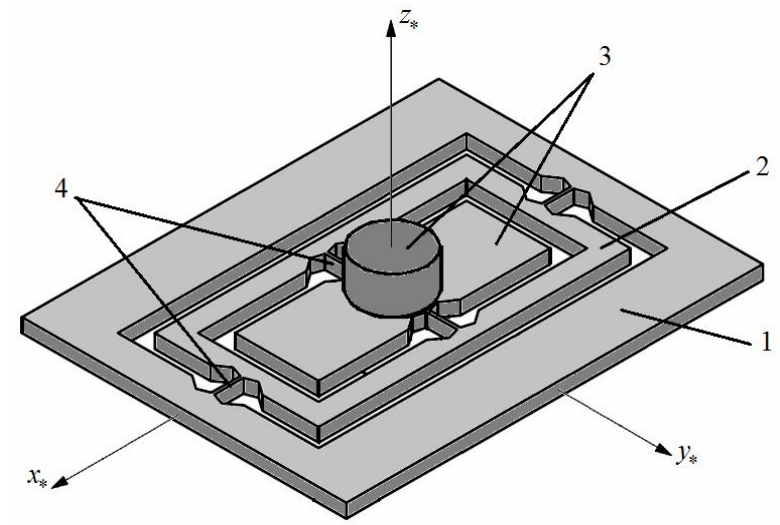

Рис. 1. Конструкционная схема прибора

Для описания положения чувствительного элемента 3 введем системы координат (рис. 2): $O x_{*} y_{*} z_{*}$ - связанную с основанием гироскопа 1 (корпусом прибора), причем ось $O z_{*}$ совпадает с осью чувствительности гироскопа; $O x_{1} y_{1} z_{1}$ - связанную с промежуточной рамкой подвеса 2; $O x y z$ - связанную с внутренней рамкой, к которой закрепляется дополнительный элемент для увеличения момента инерции подвижной части системы. Начало координат - точка $O$ находится в геометрическом центре упругого подвеса.

Учитывая, что гироскоп имеет одну ось чувствительности к угловому движению основания, будем рассматривать влияние угловой скорости основания $\Omega$ на малые колебания рамок карданова подвеса по двум обобщенным координатам - по углам $\alpha$ и $\beta$ закручивания торсионов 4.

В силу рассматриваемой кинематической схемы выражение для проекций $\omega_{2 x}, \omega_{2 y}, \omega_{2 z}$ вектора $\boldsymbol{\omega}_{2}$ угловой скорости внутренней рамки на оси Oxyz имеют вид:

$$
\begin{aligned}
& \omega_{2 x}=\dot{\alpha} \cos \beta-\Omega \cos \alpha \sin \beta, \\
& \omega_{2 y}=\dot{\beta}+\Omega \sin \alpha, \\
& \omega_{2 z}=\dot{\alpha} \sin \beta+\Omega \cos \alpha \cos \beta .
\end{aligned}
$$

Будем полагать, что конструкция системы обеспечивает бесконечную жесткость на изгиб. В расчетном случае сбалансированного ЧЭ центр масс - точка $C$ внутренней рамки и жестко закрепленной на ней дополнительной инерционной массы находится в центре подвеса и совпадает с точкой $O$. 


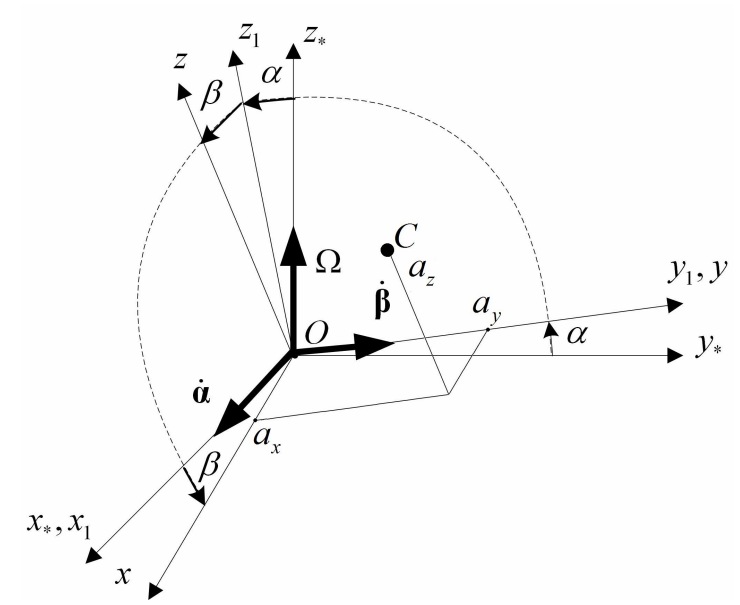

Рис. 2. Системы координат

В осях $O x_{1} y_{1} z_{1}$ зададим тензор инерции промежуточной рамки $\mathbf{J}_{1}=\operatorname{diag}\left(J_{1 x}, J_{1 y}, J_{1 z}\right)$, где $J_{1 x}, J_{1 y}, J_{1 z}-$ соответствующие осевые моменты инерции промежуточной рамки. Для расчетного случая сбалансированной внутренней пластины карданова подвеса с дополнительной инерционной массой введем тензор инерции $\mathbf{J}_{2}=\operatorname{diag}\left(J_{2 x}, J_{2 y}, J_{2 z}\right)$, где $J_{2 x}, J_{2 y}, J_{2 z}$ - осевые моменты инерции в соответствующих осях $O x y z$. Смещение инерционной массы зададим тремя постоянными компонентами $a_{x}, a_{y}, a_{z}$ вектора, соединяющего геометрический центр упругого подвеса и центр масс ЧЭ, в системе координат $O x y z$.

Составим уравнения динамики ЧЭ ММГ с помощью теоремы об изменении момента количества механической системы [0]:

$$
\frac{\tilde{d} \mathbf{K}_{2}}{d t}+\left[\boldsymbol{\omega}_{2}, \mathbf{K}_{2}\right]=\mathbf{L}_{2}, \quad \frac{\tilde{d} \mathbf{K}_{1}}{d t}+\left[\boldsymbol{\omega}_{1}, \mathbf{K}_{1}\right]=\mathbf{L}_{1},
$$

где $\mathbf{L}_{1}$ - главный момент всех внешних сил, действующих на систему «промежуточная рамка - ЧЭ», а $\mathbf{L}_{2}$ - главный момент всех внешних сил, действующих на ЧЭ. К внешним силам в данной работе относятся моменты упругости, возникающие при скручивании торсионов, и силы, характеризующие потери на внутреннее трение в материале и описываемые моделью Кельвина - Фойгта.

Момент количества движения для системы «внутренней рамки и инерционной массы» в проекциях на оси $O x y z$ имеет вид:

$$
\mathbf{K}_{2}=\left(\mathbf{J}_{2}+m_{3}\left[\begin{array}{ccc}
a_{y}^{2}+a_{z}^{2} & -a_{x} a_{y} & -a_{x} a_{z} \\
-a_{x} a_{y} & a_{x}^{2}+a_{z}^{2} & -a_{y} a_{z} \\
-a_{x} a_{z} & -a_{y} a_{z} & a_{x}^{2}+a_{y}^{2}
\end{array}\right]\right) \cdot \boldsymbol{\omega}_{2},
$$


М. Р. Сайпулаев, И. В. Меркурьев. Динамика и точность микромеханического гироскопа с учетом смещения инерционной массы

а момент количества движения для системы «промежуточная рамка ЧЭ» в проекциях на оси $O x_{1} y_{1} z_{1}$ :

$$
\mathbf{K}_{1}=\mathbf{J}_{1} \cdot \boldsymbol{\omega}_{1}+\left(\begin{array}{ccc}
\cos \beta & 0 & \sin \beta \\
0 & 1 & 0 \\
-\sin \beta & 0 & \cos \beta
\end{array}\right) \mathbf{K}_{2} .
$$

Здесь для нахождения момента инерции системы «внутренняя рамка - инерционная масса» была применена теорема Гюйгенса - Штейнеpa, a $\boldsymbol{\omega}_{1}=\left(\begin{array}{lll}\dot{\alpha} & \Omega \sin \alpha & \Omega \cos \alpha\end{array}\right)^{\mathrm{T}}$ — вектор угловой скорости промежуточной рамки в проекциях на оси $O x_{1} y_{1} z_{1}$.

Подставляя выражения (1), (3) и (4) в уравнения динамики (2) и выписывая их в проекциях на оси торсионов, получим нелинейные уравнения движения ЧЭ ММГ. При переходе от нелинейных уравнений к уравнениям малых колебаний отбросим слагаемые, характеризующие нелинейность, связанную с геометрией движения. Влияние этой нелинейности на динамику прибора исследовано в работах [5; 6].

В случае постоянной угловой скорости прибора уравнения малых колебаний имеют вид:

$$
\begin{aligned}
& \ddot{\alpha}+\omega_{\alpha}^{2} \alpha=j_{1} \Omega \dot{\beta}-\frac{\omega_{\alpha}}{Q_{\alpha}} \dot{\alpha}+\sqrt{\xi_{x} \xi_{y}} \ddot{\beta}-\left(\xi_{y}+\xi_{z}\right) \ddot{\alpha} \\
& \ddot{\beta}+\omega_{\beta}^{2} \beta=-j_{2} \Omega \dot{\alpha}-\frac{\omega_{\beta}}{Q_{\beta}} \dot{\beta}+\frac{j_{2}}{j_{1}}\left(\sqrt{\xi_{x} \xi_{y}} \ddot{\alpha}-\left(\xi_{x}+\xi_{z}\right) \ddot{\beta}\right),
\end{aligned}
$$

где введены следующие обозначения:

$$
\begin{aligned}
& j_{1}=\frac{J_{x 2}+J_{y 2}-J_{z 2}}{J_{x 1}+J_{x 2}}, \quad j_{2}=\frac{J_{x 2}+J_{y 2}-J_{z 2}}{J_{y 2}}, \\
& \xi_{x}=\frac{m_{3} a_{x}^{2}}{J_{x 1}+J_{x 2}}, \quad \xi_{y}=\frac{m_{3} a_{y}^{2}}{J_{x 1}+J_{x 2}}, \quad \xi_{z}=\frac{m_{3} a_{z}^{2}}{J_{x 1}+J_{x 2}}, \\
& \omega_{\alpha}=\sqrt{\frac{c_{\alpha}}{J_{x 1}+J_{x 2}}}, \quad \omega_{\beta}=\sqrt{\frac{c_{\beta}}{J_{y 2}}}, \quad Q_{\alpha}^{-1}=\frac{d_{\alpha}}{\omega_{\alpha}\left(J_{x 1}+J_{x 2}\right)}, \quad Q_{\beta}^{-1}=\frac{d_{\beta}}{\omega_{\beta} J_{y 2}} .
\end{aligned}
$$

Здесь $j_{1}, j_{2}$ - безразмерные массово-геометрические параметры; $\xi_{x}, \xi_{y}, \xi_{z}$ - приведенные безразмерные моменты инерции ЧЭ; $\omega_{\alpha}, \omega_{\beta}$ собственные частоты колебаний по углам $\alpha, \beta$ соответственно; $Q_{\alpha}, Q_{\beta}-$ добротность колебательного контура по углам $\alpha$ и $\beta$ соответственно; $d_{\alpha}, d_{\beta}$ - коэффициенты демпфирования; $c_{\alpha}, c_{\beta}$ - коэффициенты жесткости торсионов; $m_{3}$ - дополнительная масса. Для уменьшения демпфирования колебательного контура применяют конструкционные материалы с низким коэффициентом внутренних потерь при колебаниях, а также обеспечивают требуемую степень вакуумирования корпуса прибора. 
Анализ экспериментальных данных по измерению малых колебаний системы позволяет оценить параметры системы, в частности характерные частоты и демпфирование системы. В силу достаточно высокой добротности колебательного контура имеется возможность проводить оценку углового движения основания гироскопа в режиме свободных (неуправляемых) колебаний.

При получении уравнений колебаний (5) угловая скорость прибора полагалась малой по сравнению с собственной частотой, т. е. $|\Omega| \ll \omega_{\alpha}$.

Учитывая, что правая часть уравнений (5) представляет собой малые возмущения, т. е. $\ddot{\alpha}+\omega_{\alpha}^{2} \alpha=O(\varepsilon)$, то с указанной точностью можно записать $\ddot{\alpha}=-\omega_{\alpha}^{2} \alpha+O(\varepsilon)$. Таким образом, в правой части (5) можно избавиться от вторых производных углов $\alpha$ и $\beta$. В случае равенства собственных частот колебаний и отсутствия смещения инерционной массы уравнения (5) совпадают с известными результатами [5].

Характеристики точности ММГ существенно зависят от соотношения собственных частот $\omega_{\alpha}$ и $\omega_{\beta}$. Расчетные массово-геометрические параметры системы выбираются из условия обеспечения внутреннего резонанса в системе, т. е. при равных собственных частотах. Однако, как было указано в работах $[3 ; 4 ; 8 ; 9]$, неизвестные и непредсказуемые отклонения элементов конструкции от проектных значений, приводят к разночастотности, поэтому введем малую разночастотность $\sigma / 2$ в системе:

$$
\omega_{\alpha}=\omega_{0}, \quad \omega_{\beta}=\omega_{0}(1+\sigma / 2) .
$$

Здесь $\omega_{0}$ - характерное значение собственной частоты колебаний, принимаемое равной собственной частоте $\omega_{\alpha}$. При принятых обозначениях получим безразмерные уравнения колебаний чувствительного элемента с точностью до слагаемых первого порядка малости в виде:

$$
\begin{aligned}
& \ddot{\alpha}+\omega_{0}^{2} \alpha=j_{1} \Omega \dot{\beta}-\frac{\omega_{0} \dot{\alpha}}{Q_{\alpha}}+\omega_{0}^{2}\left(\left(\xi_{y}+\xi_{z}\right) \alpha-\sqrt{\xi_{x} \xi_{y}} \beta\right), \\
& \ddot{\beta}+\omega_{0}^{2} \beta=-j_{2} \Omega \dot{\alpha}-\frac{\omega_{0} \dot{\beta}}{Q_{\beta}}-\sigma \omega_{0}^{2} \beta+\frac{\omega_{0}^{2} j_{2}}{j_{1}}\left(\left(\xi_{x}+\xi_{z}\right) \beta-\sqrt{\xi_{x} \xi_{y}} \alpha\right) .
\end{aligned}
$$

Система уравнений динамики (7) является одночастотной системой дифференциальных уравнений с малыми возмущениями в правой части, называемой регулярно возмущенной системой [12], для исследования которой будем использовать асимптотические методы.

Решение нелинейных уравнений (7) будем искать методом осреднения Крылова - Боголюбова [12] для медленно меняющихся переменных «амплитуда - фаза» $A, B, \varphi_{1}$ и $\varphi_{2}$ :

$$
\begin{aligned}
& \alpha=A \sin \left(\omega_{0} t+\varphi_{1}\right), \quad \dot{\alpha}=A \omega_{0} \cos \left(\omega_{0} t+\varphi_{1}\right), \\
& \beta=B \sin \left(\omega_{0} t+\varphi_{2}\right), \quad \dot{\beta}=B \omega_{0} \cos \left(\omega_{0} t+\varphi_{2}\right) .
\end{aligned}
$$


М. Р. Сайпулаев, И. В. Меркурьев. Динамика и точность микромеханического гироскопа с учетом смещения инерционной массы

Проводя процедуру осреднения [12] по явно входящему времени $t$, получаем осредненную систему уравнений:

$$
\begin{aligned}
& A^{\prime}=-\frac{A}{2 Q_{a}}+\frac{j_{1} \Omega}{2 \omega_{0}} B \cos \left(\varphi_{1}-\varphi_{2}\right)+\frac{\sqrt{\xi_{x} \xi_{y}}}{2} B \sin \left(\varphi_{1}-\varphi_{2}\right), \\
& B^{\prime}=-\frac{B}{2 Q_{\beta}}-\frac{j_{2} \Omega}{2 \omega_{0}} A \cos \left(\varphi_{1}-\varphi_{2}\right)-\frac{\sqrt{\xi_{x} \xi_{y}} j_{2}}{2 j_{1}} A \sin \left(\varphi_{1}-\varphi_{2}\right), \\
& A \varphi_{1}^{\prime}=-\frac{\varepsilon\left(\xi_{y}+\xi_{z}\right)}{2} A-\frac{j_{1} \Omega}{2 \omega_{0}} B \sin \left(\varphi_{1}-\varphi_{2}\right)+\frac{\sqrt{\xi_{x} \xi_{y}}}{2} B \cos \left(\varphi_{1}-\varphi_{2}\right), \\
& B \varphi_{2}^{\prime}=\left(\frac{\sigma}{2}-\frac{\varepsilon\left(\xi_{x}+\xi_{z}\right) j_{2}}{2 j_{1}}\right) B-\frac{j_{2} \Omega}{2 \omega_{0}} A \sin \left(\varphi_{1}-\varphi_{2}\right)+\frac{\sqrt{\xi_{x} \xi_{y}} j_{2}}{2 j_{1}} A \cos \left(\varphi_{1}-\varphi_{2}\right) .
\end{aligned}
$$

Штрихом в (9) обозначено дифференцирование по безразмерному времени $\tau=\omega_{0} t$. Полученная модель в линейном приближении описывает свободные колебания ЧЭ гироскопа на подвижном основании.

\section{2 Решение уравнений колебаний в частном случае}

Рассмотрим случай расположения центра масс инерционной массы на одной из осей торсионов, т. е. $a_{x}=0$ или $a_{y}=0$. Указанный случай представляет интерес из-за возможности получения аналитического решения системы дифференциальных уравнений (9). Полученные решения могут быть использованы для построения методик идентификации параметров, а также оценки ухода гироскопа и его учета с помощью методики алгоритмической компенсации. При таком допущении система уравнений при неподвижном основании $(\Omega=0)$ примет вид:

$$
\begin{array}{ll}
A^{\prime}=-\frac{1}{2 Q_{a}} A, & A \varphi_{1}^{\prime}=-\frac{1}{2}\left(\xi_{y}+\xi_{z}\right) A, \\
B^{\prime}=-\frac{1}{2 Q_{\beta}} B, & B \varphi_{2}^{\prime}=\frac{1}{2}\left(\sigma-\frac{j_{2}}{j_{1}}\left(\xi_{x}+\xi_{z}\right)\right) B .
\end{array}
$$

Решение этой системы можно записать в виде:

$$
\begin{array}{ll}
A(\tau)=A(0) \exp \left(-\frac{1}{2 Q_{\alpha}} \tau\right), & \varphi_{1}(\tau)=-\frac{1}{2}\left(\xi_{y}+\xi_{z}\right) \tau+\varphi_{1}(0), \\
B(\tau)=B(0) \exp \left(-\frac{1}{2 Q_{\beta}} \tau\right), & \varphi_{2}(\tau)=\frac{1}{2}\left(\sigma-\frac{j_{2}}{j_{1}}\left(\xi_{x}+\xi_{z}\right)\right) \tau+\varphi_{2}(0), \\
\text { где } A_{0}=A(0), B_{0}=B(0), \varphi_{10}=\varphi_{1}(0), \varphi_{20}=\varphi_{2}(0)-\text { начальные условия. }
\end{array}
$$
Возвращаясь к исходным переменным, решение примет вид: 


$$
\begin{aligned}
& \alpha(t)=A(0) \exp \left(-\frac{\omega_{0}}{2 Q_{\alpha}} t\right) \sin \left(\omega_{0}\left(1-\frac{1}{2}\left(\xi_{y}+\xi_{z}\right)\right) t+\varphi_{1}(0)\right) \\
& \beta(t)=B(0) \exp \left(-\frac{\omega_{0}}{2 Q_{\beta}} t\right) \sin \left(\omega_{0}\left(1+\frac{\sigma}{2}-\frac{j_{2}}{2 j_{1}}\left(\xi_{x}+\xi_{z}\right)\right) t+\varphi_{2}(0)\right) .
\end{aligned}
$$

Таким образом, смещение инерционной массы из центра подвеса приводит к расщеплению собственных частот системы. Полученное аналитическое решение может быть использовано при обработке стендовых испытаний прибора.

\section{3 Сравнение результатов моделирования с экспериментом}

Для оценки адекватности построенной модели сравним результаты моделирования с экспериментальными данными. В качестве измерительной информации, получаемой из системы наблюдения, имеем медленно меняющиеся переменные Ван-дер-Поля $p_{1}, q_{1}, p_{2}, q_{2}$ [5], которые связаны с углами $\alpha$ и $\beta$ соотношениями:

$$
\alpha=p_{1} \sin \left(\omega_{0} t\right)+q_{1} \cos \left(\omega_{0} t\right), \quad \beta=p_{2} \sin \left(\omega_{0} t\right)+q_{2} \cos \left(\omega_{0} t\right) .
$$

Получение информации осуществляется с помощью электростатических датчиков. Учитывая, что решения получены в переменных «амплитуда - фаза», то для их сравнения необходимо пересчитать амплитуды по экспериментальным данным по формулам:

$$
A=\sqrt{p_{1}^{2}+q_{1}^{2}}, \quad B=\sqrt{p_{2}^{2}+q_{2}^{2}},
$$

а для обратного перехода справедливы соотношения:

$$
p_{1}=A \cos \varphi_{1}, \quad q_{1}=A \sin \varphi_{1}, \quad p_{2}=B \cos \varphi_{2}, \quad q_{2}=B \sin \varphi_{2} .
$$

Экспериментальные данные были получены для прибора со следующими числовыми значениями параметров математической модели:

$$
\begin{aligned}
& Q_{\alpha}=3856, \quad Q_{\beta}=3938, \quad j_{1}=1.35 \cdot 10^{-3}, \quad j_{2}=1.46 \cdot 10^{-3}, \\
& \left(\xi_{y}+\xi_{z}\right)=0.2 \cdot 10^{-5}, \quad\left(\frac{\sigma}{2}-\frac{j_{2}}{2 j_{1}}\left(\xi_{x}+\xi_{z}\right)\right)=3 \cdot 10^{-5},
\end{aligned}
$$

а начальные условия были приняты равными значениям переменных Вандер-Поля из измерения в начальный момент времени:

$$
\begin{aligned}
& p_{10}=13.467 \cdot 10^{-3}, \quad q_{10}=20.429 \cdot 10^{-3}, \quad A_{0}=24.468 \cdot 10^{-3}, \quad \varphi_{10}=0.988, \\
& p_{20}=0.787 \cdot 10^{-3}, \quad q_{20}=1.172 \cdot 10^{-3}, \quad B_{0}=1.412 \cdot 10^{-3}, \quad \varphi_{20}=0.978 .
\end{aligned}
$$

Сравним поведение зависимостей амплитуд $A$ и $B$ от безразмерного времени $\tau$, которые приведены на рис. 3. Как видно по рис. 3 демпфирование колебаний в эксперименте соответствует решению (11). А учет явления разнодобротности $\left(Q_{\alpha} \neq Q_{\beta}\right)$ обеспечивает близость зависимостей каждой из амплитуд к соответствующим экспериментальным данным. На рис. 3-5 цифрой «1» обозначены зависимости, полученные по результатам моделирования, а цифрой «2» - экспериментальные данные. 
М. Р. Сайпулаев, И. В. Меркурьев. Динамика и точность микромеханического гироскопа с учетом смещения инерционной массы

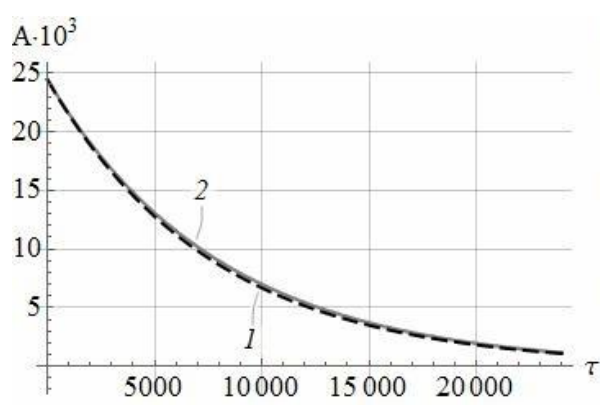

a)

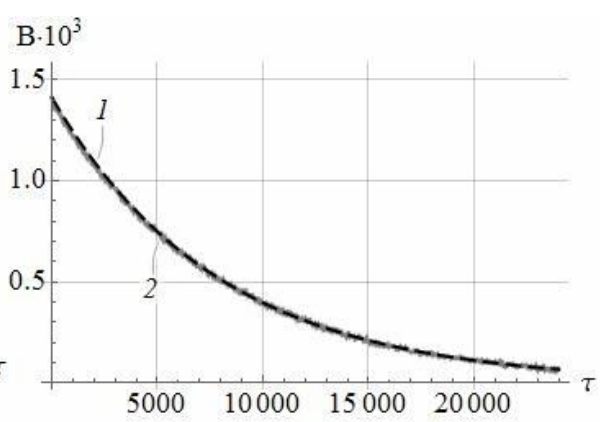

б)

Рис. 3. Зависимости амплитуд $A$ и $B$ от безразмерного времени $\tau$

При проверке решения для медленно изменяющихся фаз (11) возникают трудности с вычислением значений обратных тригонометрических функций для зашумленных экспериментальных данных. Поэтому проверку поведения для фаз будем проводить сравнением зависимостей переменных Ван-дер-Поля от времени. При этом для получения аналитических выражений для $p_{1}, q_{1}, p_{2}, q_{2}$, соответствующих решению (11), в формулы перехода (15) будет подставлено решение для переменных «амплитуда - фаза». В результате графики зависимостей медленно меняющихся переменных Ван-дер-Поля представлены на рис. 4.

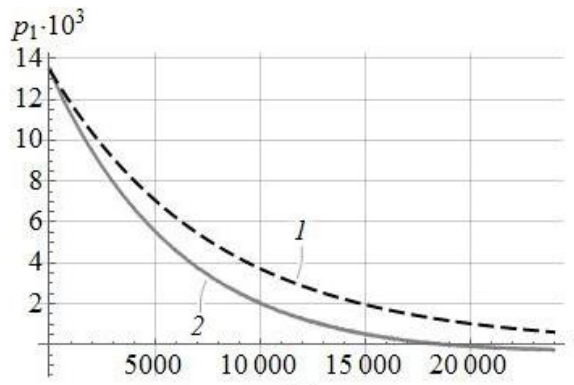

a)

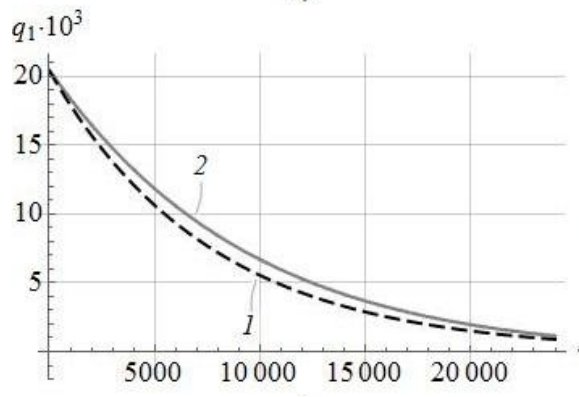

B)

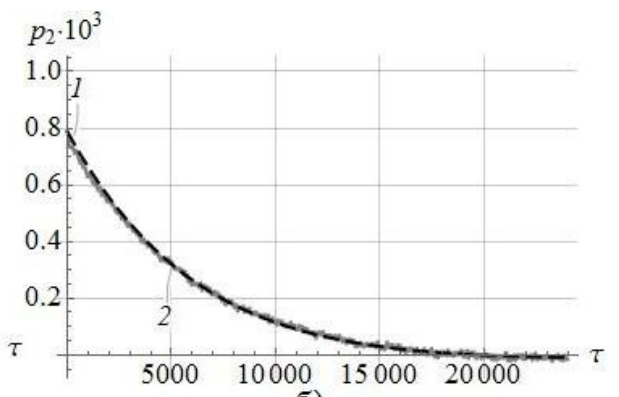

б)

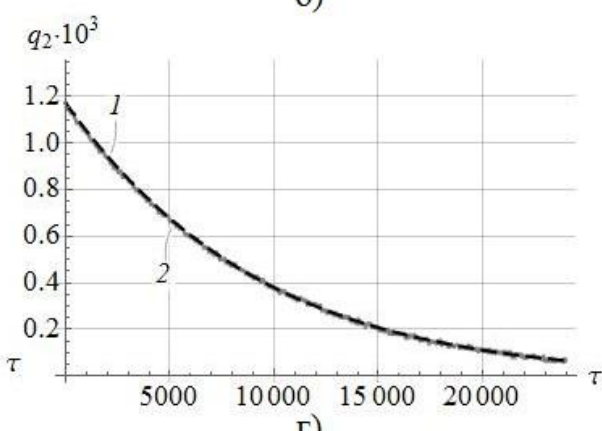

г)

Рис. 4. Зависимости переменных Ван-дер-Поля $p_{1}, q_{1}, p_{2}, q_{2}$ от безразмерного времени $\tau$ 
По графикам рис. 4 видно, что для переменных $p_{2}, q_{2}$ имеем достаточно точное совпадение результатов моделирования и экспериментальных данных. Для переменных $p_{1}, q_{1}$ наблюдается небольшое расхождение в числовых значениях при качественном совпадении зависимостей. Эти расхождения могут быть объяснены нелинейными эффектами, в частности, упомянутой выше геометрической нелинейностью [5; 6]. Учет нелинейных эффектов при построении математических моделей ММГ, увеличивает точность приборов и, как следствие, инерциальных навигационных систем на их основе $[1 ; 2 ; 6]$.

Для оценки ухода гироскопа, связанного с нелинейными или побочными эффектами, введем вспомогательный функционал $I$, характеризующий угол прецессии орбиты колебаний ЧЭ [5; 10]:

$$
I=\frac{2 \sqrt{j_{1} j_{2}}\left(q_{1} q_{2}+p_{1} p_{2}\right)}{j_{2}\left(q_{1}^{2}+p_{1}^{2}\right)-j_{1}\left(q_{2}^{2}+p_{2}^{2}\right)}=\frac{2 \sqrt{j_{1} j_{2}} A B \cos \left(\varphi_{2}-\varphi_{1}\right)}{j_{2} A^{2}-j_{1} B^{2}},
$$

для которого справедливо соотношение:

$$
\theta=\frac{1}{2} \arctan I
$$

где $\theta=-K \int_{0}^{\tau} \Omega\left(\tau_{1}\right) d \tau_{1}-$ угол, пропорциональный интегралу от угловой скорости, а $K=\sqrt{j_{1} j_{2}} / 2-$ масштабный коэффициент.

При подстановке решения (11) в выражение (16) получим:

$$
I=\frac{4 K A_{0} B_{0} \exp \left(-\frac{Q_{\alpha}^{-1}+Q_{\beta}^{-1}}{2} \tau\right) \cos \left(\left[\sigma-\left(\frac{j_{2}\left(\xi_{x}+\xi_{z}\right)}{j_{1}}-\xi_{y}-\xi_{z}\right)\right] \frac{\tau}{2}+\Delta \varphi_{0}\right)}{j_{2} A_{0}^{2} \exp \left(-Q_{\alpha}^{-1} \tau\right)-j_{1} B_{0}^{2} \exp \left(-Q_{\beta}^{-1} \tau\right)} .
$$

Здесь $\Delta \varphi_{0}=\varphi_{20}-\varphi_{10}-$ разность фаз в начальный момент времени.

На рис. 5 представлена зависимость расчетного (1) и экспериментального (2) значения функционала $I$ от безразмерного времени $\tau$. Анализ значений функционала $I$, рассчитанного по экспериментальным данным, показывает на существенную зависимость функционала от амплитуды колебаний и шумов измерений. При уменьшении амплитуды колебаний отношение полезного сигнала к шуму измерений уменьшается и, соответственно, увеличиваются погрешности в определении ухода гироскопа. 
М. Р. Сайпулаев, И. В. Меркурьев. Динамика и точность микромеханического гироскопа с учетом смещения инерционной массы

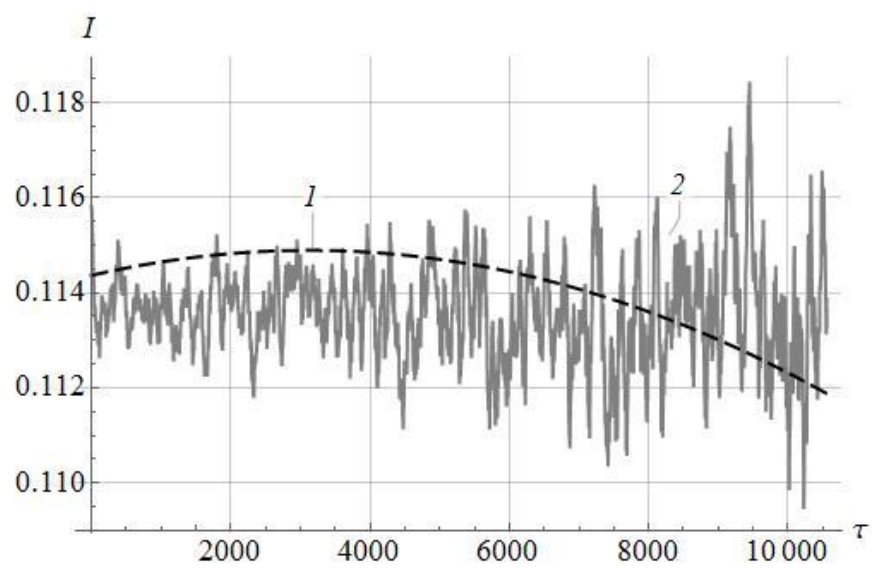

Рис. 5. Зависимость функционала $I$ от безразмерного времени

Точность построенной модели оценим по относительным погрешностям амплитуд колебаний $\delta A$ и $\delta B$, которые будем рассчитывать по формулам:

$$
\delta A=\left|\left(A_{e}-A_{m}\right) / A_{m}\right| \cdot 100 \%, \quad \delta B=\left|\left(B_{e}-B_{m}\right) / B_{m}\right| \cdot 100 \% .
$$

Здесь нижнему индексу «е» соответствуют значения амплитуд, рассчитанные по экспериментальным данным; а индексу «m» - значения амплитуд, полученные по решению (11). Графики зависимостей соответствующих относительных погрешностей от безразмерного времени представлены на рис. 6.

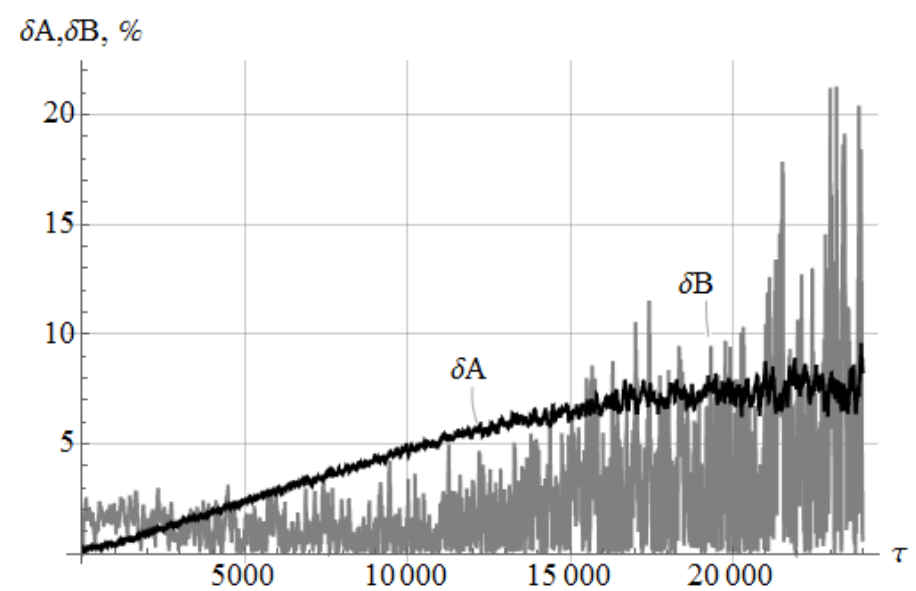

Рис. 6. Зависимости относительных погрешностей для амплитуд $\delta A$ и $\delta B$ колебаний

Из графиков рис. 6 видно, что относительная погрешность для амплитуды $\delta A$ не превышает $8,75 \%$, а для амплитуды $\delta B-21,3 \%$. 
Полученные значения характеризуют адекватность построенной математической модели малых колебаний ЧЭ. Уточнение параметров математической модели с помощью методики идентификации параметров позволит увеличить точность ММГ путем алгоритмической компенсации систематического ухода гироскопа, вызванного разнодобротностью, разночастотностью и смещением инерционной массы относительно геометрического центра упругого подвеса.

\section{Заключение}

Построенная новая математическая модель ММГ R-R типа описывает динамику гироскопа на подвижном основании с учетом инструментальных погрешностей изготовления - малой неравножесткости и разнодобротности упругого подвеса, а также малого смещения центра масс чувствительного элемента относительно центра упругого подвеса. Проведенный сравнительный анализ результатов математического моделирования с экспериментальными данными подтверждает адекватность разработанной модели колебаний ЧЭ ММГ.

Результаты работы могут быть использованы для повышения точности прибора с помощью алгоритма аналитической компенсации систематических погрешностей гироскопа.

\section{Литература}

1. Пешехонов В. Г. Современное состояние перспективы развития гироскопических систем // Гироскопия и навигация. 2011. № 1. С. 3-16.

2. Журавлев В. Ф. Управляемый маятник Фуко как модель одного класса свободных гироскопов // Изв. РАН. МТТ. 1997. № 6. С. 27-35.

3. Распопов В. Я. Микромеханические приборы: учеб. пособие. М.: Машиностроение, 2007. $400 \mathrm{c}$.

4. Басараб М. А., Кравченко В. Ф., Матвеев В. А. Методы моделирования и цифровая обработка сигналов в гироскопии. М.: Физматлит, 2008. 248c.

5. Меркурьев И. В., Подалков В. В. Динамика микромеханического и волнового твердотельного гироскопов. М.: Физматлит, 2009. 228 с.

6. Меркурьев И. В., Подалков В. В. Нелинейные эффекты в динамике микромеханического гироскопа // Вестник МЭИ. 2004. № 2. С. 5-10.

7. Basarab M., Lunin B., Vakhlyarskiy D., Chumankin E. Investigation of Nonlinear High-Intensity Dynamic Processes in a Non-ideal Solid-State Wave Gyroscope Resonator // 27th Saint Petersburg International Conference on Integrated Navigation Systems, ICINS 2020 - Proceedings, № 9133943.

8. Basarab M. A., Matveev V. A., Lunin B. S., Fetisov S. V. Influence of nonuniform thickness of hemispherical resonator gyro shell on its unbalance parameters // Gyroscopy and Navigation. 2018. № 8 (2). P. 97-103.

9. Apostolyuk V. Theory and Design of Micromechanical Vibratory Gyroscopes // MEMS/NEMS Handbook. 2006. № 1(6). C. 173-195.

10. Askari S., Asadian M. H. and Shkel A. M. High quality factor MEMS gyroscope with whole angle mode of operation // 2018 IEEE Int. Symposium on Inertial Sensors and Systems (INERTIAL). Moltrasio, Italy, 2018. P. 1-4. 
М. Р. Сайпулаев, И. В. Меркурьев. Динамика и точность микромеханического гироскопа с учетом смещения инерционной массы

11. Маркеев А. П. Теоретическая механика: учебник для высших учебных заведений. М., Ижевск: Регулярная и хаотическая механика, 2007. 592 с.

12. Боголюбов Н. Н., Митропольский Ю. А. Асимптотические методы в теории нелинейных колебаний. М.: Наука, 1974. 503 с.

\title{
DYNAMICS AND ACCURACY OF A MICROMECHANICAL GYROSCOPE WITH PROVISION FOR THE DISPLACEMENT OF INERTIAL MASS
}

\author{
Musa R. Saipulaev \\ Research Assistant, \\ National Research University "Moscow Power Engineering Institute" \\ 14 Krasnokazarmennaya St., Moscow 111250, Russia \\ saypulaevmr@mail.ru
}

Igor V. Merkuryev

Dr. Sci. (Engineering), A/Prof.,

Head of Department of Robotics, Mechatronics, Dynamics and Machine Strength

National Research University "Moscow Power Engineering Institute"

14 Krasnokazarmennaya St., Moscow 111250, Russia

nir4s@ya.ru

Abstract. We consider a micromechanical gyroscope (MMG), used in navigation and motion control systems for moving objects. In order to improve the accuracy of the gyroscope in the integrating mode of operation on a rocking base, we have studied the dynamics and accuracy of the gyroscope, taking into account the small instrumental errors in creation of the sensitive element of the gyroscope - unequal rigidity of the elastic support elements, small shifts of the gyroscope center of mass relative to the geometric center of the assembly. The method for increasing the accuracy of the gyroscope is based on the construction of a new mathematical model of its dynamics and errors using the general theorems of dynamics and Krylov-Bogolyubov averaging methods. The new mathematical model of oscillations of the sensitive element of a weight-size prototype makes it possible to estimate the errors of the gyroscope in the integrating mode of operation in form of dependence of the precession angle on the parameters of the differences in Q factor, frequency and shifts of the inertial mass on a rocking base of the device. The article presents a comparative analysis of the constructed model with experimental data obtained for the case of free oscillations of the sensitive element of the gyroscope on a fixed base. Based on the results of analysis, we have confirmed the adequacy of the constructed mathematical model of a weight-size prototype. We propose to use the methods of parameter identification for matching the experimental data with the simulation results. It is shown that the shifts of the inertial mass leads to a change in the natural frequencies of the gyroscope oscillations and the additional different frequencies. The results of the work can be used to improve the accuracy of the device using the algorithm for analytical compensation of the gyro error.

Keywords: RR-type gyroscope; gyroscopic precession; gyroscope error estimation; micromechanical gyroscope; free oscillations. 


\section{References}

1. Peshekhonov V. G. Sovremennoe sostoyanie perspektivy razvitiya giroskopicheskikh sistem [Current State of the Prospects for the Development of Gyroscopic Systems]. Gyroscopy and Navigation. 2011. No. 1. Pp. 3-16.

2. Zhuravlev V. F. Upravlyaemyi mayatnik Fuko kak model odnogo klassa svobodnykh giroskopov [Controlled Foucault Pendulum as a Model of One class of Free Gyroscopes]. Mechanics of Solids. 1997. No. 6. Pp. 27-35.

3. Raspopov V. Ya. Mikromekhanicheskie pribory [Micromechanical Devices]. Moscow: Mashinostroyeniye Publ., 2007. 400 p.

4. Basarab M. A., Kravchenko V. F., Matveev V. A. Metody modelirovaniya i tsifrovaya obrabotka signalov $v$ giroskopii [Simulation Methods and Digital Signal Processing in Gyroscopy]. Moscow: Fizmatlit Publ., 2008. 248 p.

5. Merkuryev I. V., Podalkov V. V. Dinamika mikromekhanicheskogo i volnovogo tverdotelnogo giroskopov [Dynamics of Micromechanical and Wave Solid-State Gyroscopes]. Moscow: Fizmatlit Publ., 2009. 228 p.

6. Merkuryev I. V., Podalkov V. V. Nelineinye effekty v dinamike mikromekhanicheskogo giroskopa [Nonlinear Effects in the Dynamics of a Micromechanical Gyroscope]. Vestnik MEI. 2004. No. 2. Pp. 5-10.

7. Basarab M., Lunin B., Vakhlyarskiy D., Chumankin E. Investigation of Nonlinear High-Intensity Dynamic Processes in a Non-Ideal Solid-State Wave Gyroscope Resonator. $27^{\text {th }}$ Saint Petersburg International Conference on Integrated Navigation Systems, ICINS 2020 Proceedings. No. 9133943.

8. Basarab M. A., Matveev V. A., Lunin B. S., Fetisov S. V. Influence of Nonuniform Thickness of Hemispherical Resonator Gyro Shell on its Unbalance Parameters. Gyroscopy and Navigation. 2018. No.8 (2). Pp. 97-103.

9. Apostolyuk V. Theory and Design of Micromechanical Vibratory Gyroscopes. MEMS/NEMS Handbook. 2006. No. 1(6). Pp. 173-195.

10. Askari S., Asadian M. H., Shkel A. M. High Quality Factor MEMS Gyroscope with Whole Angle Mode of Operation. 2018 IEEE Int. Symposium on Inertial Sensors and Systems (INERTIAL). Moltrasio, Italy, 2018. Pp. 1-4.

11. Markeyev A. P. Teoreticheskaya mekhanika [Theoretical Mechanics]. Moscow; Izhevsk: Regulyarnaya i khaoticheskaya mekhanika Publ., 2007. 592 p.

12. Bogolyubov N. N., Mitropolskiy Yu. A. Asimptoticheskie metody v teorii nelineinykh kolebanii [Asymptotic Methods in the Theory of Nonlinear Oscillations]. Moscow: Nauka Publ., 1974. 503 p.

Статья поступила в редакцию 04.11.2020; одобрена после рецензирования 30.11.2020; принята к публикации 10.12.2020. 